\title{
Influence of calcium and alumina-based pozzolanas on the strength properties of low calcium fly ash geopolymer concrete
}

\author{
$K$ Saiteja Chary ${ }^{*}, S$ Shrihari ${ }^{2}, V$ Siva Prasad Raju ${ }^{3}$, and Srinivasa Reddy $\mathrm{V}^{4}$ \\ ${ }^{1} \mathrm{M}$. Tech (Structural Engineering), Department of Civil Engineering, VJIT, Hyderabad, India. \\ ${ }^{2}$ Professor of Civil Engineering, VJIT, Hyderabad, India. \\ ${ }^{3}$ Assistant Professor of Civil Engineering, GRIET, Hyderabad, India. \\ ${ }^{4}$ Professor of Civil Engineering, GRIET, Hyderabad, India.
}

\begin{abstract}
This work presents the effect of Ground granulated blast furnace slag (GGBS), fly ash (FA) and metakaolin (MK) on the strength properties of geopolymer concrete (GPC). Geopolymer concrete made with FA produces calcium aluminosilicate hydrate (C-A-S-H) product due to presence of alumina and sodium aluminosilicate hydrate (N-A-S-H) gel as main reaction product of polymerization. Geopolymer concrete made with FA and GGBS, calcium silicate hydrate (C-S$\mathrm{H})$ also gets produced additionally with calcium aluminosilicate hydrate (C-A-S-H) gel and sodium aluminosilicate hydrate (N-A-S-H) gel due to presence of high content of $\mathrm{CaO}$ in GGBS. This additional product imparts more strength performance in GPC. In geopolymer concrete made with FA and MK, the more amount of calcium aluminosilicate hydrate (C-A-S-H) is produced due to presence of high amount of alumina in metakaolin along with sodium aluminosilicate hydrates (N-A-S-H) giving more strength to GPC. Metakaolin is recommended to be used for the development of GPC because it has high amount of alumina.
\end{abstract}

\section{Introduction}

The processing of one ton of cement releases one ton of "CO2" carbon dioxide into the atmosphere contributing to global warming [1]. A prerequisite for the current situation is that we create additional cement production facilities or look for alternative binder systems to manufacture concrete? In the other case, a large amount of fly ash is generated from thermal power plants around the world and is typically used as filler in low-level zones [2]. "Geopolymer concrete" is an alternative binding system with fly ash for concrete removal. Geopolymer has the optimal characteristics of rock shaping materials ${ }^{1}$, i.e. toughness, chemical inertness, and durability as a type of amorphous aluminium hydroxide substance [3]. In combination with Geopolymer binding aggregates, Geopolymer composites are made. Ideologies as they have very strong early strength, they are suitable for the design,

\footnotetext{
* Corresponding author: kashisaitejachary@gmail.com
}

repair, and pre-casting of infrastructure, they can monitor their set times and stay intact very long without any repair [4]. Geo-polymer properties involve low shrinkage, freeze thaw resistance, high early strength, corrosion \& sulphate resistance [5]. These greater-alkaline additives do not cause an aggregate alkaline reaction. The binder is a controversial substance of low carbon dioxide. It's doesn't depend on the limestone calcinations that emits carbon dioxide. This innovation will save 80 percent of the production of carbon dioxide from the cement and concrete sectors. This work presents the effect of Ground granulated blast furnace slag (GGBS), fly ash (FA) and metakaolin (MK) on the strength properties of geopolymer concrete (GPC)

\section{Mix proportions}

\subsection{Geopolymer Concrete}

The mix ingredients of geopolymer concrete are-

1. $16 \mathrm{M} \mathrm{NaOH}$

2. $\mathrm{SiO}_{2} / \mathrm{Na}_{2} \mathrm{O}=2.0$ 
3. $\mathrm{Na}_{2} \mathrm{SiO}_{3} / \mathrm{NaOH}=2.5$

4. Fly Ash $=450 \mathrm{~kg} / \mathrm{m}^{3}$

5. Alkali Activator solution (AAS) / Fly ash $=0.50$

6. Fine aggregate $=505 \mathrm{~kg} / \mathrm{m}^{3}$

7. $20 \mathrm{~mm}$ Coarse aggregate $=1246 \mathrm{~kg} / \mathrm{m}^{3}$

8. $60^{\circ} \mathrm{C}$ temperature oven heat cured for $24 \mathrm{~h}$ period

9. Rest period $=0$ days (casted cubes are immediately sealed and kept in oven with moulds on)

\subsection{Conventional Concrete}

The mix proportions for conventional concrete are-

1. Cement $=450 \mathrm{~kg} / \mathrm{m}^{3}$

2. Water $/$ cement ratio $=0.50$

3. Fine aggregate $=505 \mathrm{~kg} / \mathrm{m}^{3}$

4. $20 \mathrm{~mm}$ Coarse aggregate $=1246 \mathrm{~kg} / \mathrm{m}^{3}$

5. Water $=225 \mathrm{l} / \mathrm{m}^{3}$

\section{Compressive Strength}

This investigation is carried out to study the compressive strength of conventional and Geopolymer concrete The compression test on cubes and cylinders were conducted according to Indian
Standard specifications (IS: 516 - 1959). For the given mixture proportion casted concrete cubes of $150 \mathrm{~mm} \times 150 \mathrm{~mm} \times 150 \mathrm{~mm}$ and Cylinder of $150 \mathrm{~mm}$ Diameter and $300 \mathrm{~mm}$ Height provided with oven curing temperature and ambient temperature.

\section{Split tensile Strength}

The split tensile strength test was carried out on the compression testing machine. The casting and testing of the specimens were done as per IS 5816: 1999. This investigation was carried out to study the split tensile strength of conventional and Geopolymer concrete. Cylinders of size $150 \times 300 \mathrm{~mm}$ are cast and tested to study the split tensile strength as per IS:5816 - 1999. From the following table it can be observed that the GGBS has high $\mathrm{CaO}$ and Silica content and moderate amount of alumina oxide whereas FA has high amount of silica and alumina oxides. Metakaolin has very high amount of silica and Alumina. Silica and alumina are the major requirements for polymerization. Table 2-4 present the ingredients of GPC made with FA, FA+GGBS and $\mathrm{FA}+\mathrm{MK}$

Table 1. Chemical composition of GGBS, FA and MK

\begin{tabular}{|c|c|c|c|}
\hline Chemical composition & GGBS \% by mass & FA \% by mass & MK \% by mass \\
\hline $\mathrm{SiO}_{2}$ & 43.4 & 62.10 & 53.20 \\
\hline $\mathrm{Al}_{2} \mathrm{O}_{3}$ & 12.5 & 27.44 & 43.90 \\
\hline $\mathrm{Fe}_{2} \mathrm{O}_{3}$ & 0.3 & 4.57 & 0.38 \\
\hline $\mathrm{CaO}$ & 40.0 & 0.83 & 0.02 \\
\hline $\mathrm{MgO}$ & 1.5 & 0.55 & 0.05 \\
\hline $\mathrm{SO}_{3}$ & - & 0.40 & - \\
\hline $\mathrm{Na}_{2} \mathrm{O}$ & 0.9 & 0.04 & 0.17 \\
\hline $\mathrm{K}_{2} \mathrm{O}$ & 0.6 & 1.17 & 0.10 \\
\hline
\end{tabular}

Table 2. Ingredients of GPC made with Fly ash

\begin{tabular}{|c|c|c|c|c|}
\hline GPC Mix Designation & $\begin{array}{c}\mathrm{NaOH} \\
\text { Molarity }\end{array}$ & $\begin{array}{c}\mathrm{Na}_{2} \mathrm{SiO}_{3} / \\
\mathrm{NaOH} \text { ratio }\end{array}$ & $\begin{array}{c}\mathrm{SiO}_{2} / \mathrm{Na}_{2} \mathrm{O} \\
\text { ratio }\end{array}$ & $\begin{array}{c}\mathrm{AAS} / \\
\text { Fly ash }\end{array}$ \\
\hline GP1 & $8 \mathrm{M}$ & 2.5 & 2.0 & 0.5 \\
\hline GP2 & $10 \mathrm{M}$ & 2.5 & 2.0 & 0.5 \\
\hline GP3 & $12 \mathrm{M}$ & 2.5 & 2.0 & 0.5 \\
\hline GP4 & $14 \mathrm{M}$ & 2.5 & 2.0 & 0.5 \\
\hline GP5 & $16 \mathrm{M}$ & 2.5 & 2.0 & 0.5 \\
\hline GP6 & $18 \mathrm{M}$ & 2.5 & 2.0 & 0.5 \\
\hline
\end{tabular}

Table 3. Ingredients of GPC made with Fly ash and GGBS

\begin{tabular}{|c|c|c|c|c|}
\hline GPC Mix Designation & $\begin{array}{c}\mathrm{NaOH} \\
\text { Molarity }\end{array}$ & $\begin{array}{c}\mathrm{Na}_{2} \mathrm{SiO}_{3} / \\
\mathrm{NaOH} \text { ratio }\end{array}$ & $\begin{array}{c}\mathrm{SiO}_{2} / \mathrm{Na}_{2} \mathrm{O} \\
\text { ratio }\end{array}$ & $\begin{array}{c}\text { AAS / } \\
\text { (Fly ash } \\
\text { +GGBS) }\end{array}$ \\
\hline GP7 & $8 \mathrm{M}$ & 2.5 & 2.0 & 0.5 \\
\hline GP8 & $10 \mathrm{M}$ & 2.5 & 2.0 & 0.5 \\
\hline GP9 & $12 \mathrm{M}$ & 2.5 & 2.0 & 0.5 \\
\hline GP10 & $14 \mathrm{M}$ & 2.5 & 2.0 & 0.5 \\
\hline
\end{tabular}




\begin{tabular}{|c|c|c|c|c|}
\hline GP11 & $16 \mathrm{M}$ & 2.5 & 2.0 & 0.5 \\
\hline GP12 & $18 \mathrm{M}$ & 2.5 & 2.0 & 0.5 \\
\hline
\end{tabular}

Table 4. Ingredients of GPC made with Fly ash and MK

\begin{tabular}{|c|c|c|c|c|}
\hline GPC Mix Designation & $\begin{array}{c}\mathrm{NaOH} \\
\text { Molarity }\end{array}$ & $\begin{array}{c}\mathrm{Na}_{2} \mathrm{SiO}_{3} / \\
\mathrm{NaOH} \text { ratio }\end{array}$ & $\begin{array}{c}\mathrm{SiO}_{2} / \mathrm{Na}_{2} \mathrm{O} \\
\text { ratio }\end{array}$ & $\begin{array}{c}\text { AAS / } \\
\text { (Fly ash +MK) }\end{array}$ \\
\hline GP13 & $8 \mathrm{M}$ & 2.5 & 2.0 & 0.5 \\
\hline GP14 & $10 \mathrm{M}$ & 2.5 & 2.0 & 0.5 \\
\hline GP15 & $12 \mathrm{M}$ & 2.5 & 2.0 & 0.5 \\
\hline GP16 & $14 \mathrm{M}$ & 2.5 & 2.0 & 0.5 \\
\hline GP17 & $16 \mathrm{M}$ & 2.5 & 2.0 & 0.5 \\
\hline GP18 & $18 \mathrm{M}$ & 2.5 & 2.0 & 0.5 \\
\hline
\end{tabular}

Table 5. $\mathrm{NaOH}$ flakes to be added to make One litre of $\mathrm{NaOH}$ solution

\begin{tabular}{|c|c|}
\hline Molarity NaOH flakes & $\begin{array}{c}\text { NaOH flakes to be added to make } \\
\text { One litre of NaOH solution (grams) }\end{array}$ \\
\hline $8 \mathrm{M}$ & 296 \\
\hline $10 \mathrm{M}$ & 364 \\
\hline $12 \mathrm{M}$ & 429 \\
\hline $14 \mathrm{M}$ & 491 \\
\hline $16 \mathrm{M}$ & 552 \\
\hline $18 \mathrm{M}$ & 610 \\
\hline
\end{tabular}

Table 6. Mix quantities for GPC made with FA

\begin{tabular}{|c|c|c|c|c|c|c|}
\hline \multirow{2}{*}{$\begin{array}{l}\text { GPC } \\
\text { Mix }\end{array}$} & \multicolumn{2}{|c|}{$\operatorname{AAS}\left(\mathrm{kg} / \mathrm{m}^{3}\right)$} & \multirow{2}{*}{$\begin{array}{l}\text { Fly ash } \\
\left(\mathrm{kg} / \mathrm{m}^{3}\right)\end{array}$} & \multirow{2}{*}{$\begin{array}{c}\text { Fine } \\
\text { Aggregate } \\
\left(\mathrm{kg} / \mathrm{m}^{3}\right)\end{array}$} & \multirow{2}{*}{$\begin{array}{c}\text { Coarse } \\
\text { Aggregate } \\
\left(\mathrm{kg} / \mathrm{m}^{3}\right)\end{array}$} & \multirow{2}{*}{ Curing type } \\
\hline & $\mathrm{NaOH}$ & $\mathrm{Na}_{2} \mathrm{SiO}_{3}$ & & & & \\
\hline GP1 & 64.29 & 160.71 & 450 & 505 & 1246 & $\begin{array}{c}\text { Heat @ } 60{ }^{\circ} \mathrm{C} \text { for } 24 \mathrm{~h} \text { exposure } \\
\text { Sun dried for } 28 \text { days }\end{array}$ \\
\hline GP2 & 64.29 & 160.71 & 450 & 505 & 1246 & $\begin{array}{c}\text { Heat @ } 60{ }^{\circ} \mathrm{C} \text { for } 24 \mathrm{~h} \text { exposure } \\
\text { Sun dried for } 28 \text { days }\end{array}$ \\
\hline GP3 & 64.29 & 160.71 & 450 & 505 & 1246 & $\begin{array}{c}\text { Heat @ } 60{ }^{\circ} \mathrm{C} \text { for } 24 \mathrm{~h} \text { exposure } \\
\text { Sun dried for } 28 \text { days }\end{array}$ \\
\hline GP4 & 64.29 & 160.71 & 450 & 505 & 1246 & $\begin{array}{c}\text { Heat @ } 60^{\circ} \mathrm{C} \text { for } 24 \mathrm{~h} \text { exposure } \\
\text { Sun dried for } 28 \text { days }\end{array}$ \\
\hline GP5 & 64.29 & 160.71 & 450 & 505 & 1246 & $\begin{array}{c}\text { Heat @ } 60{ }^{\circ} \mathrm{C} \text { for } 24 \mathrm{~h} \text { exposure } \\
\text { Sun dried for } 28 \text { days }\end{array}$ \\
\hline GP6 & 64.29 & 160.71 & 450 & 505 & 1246 & $\begin{array}{c}\text { Heat @ } 60{ }^{\circ} \mathrm{C} \text { for } 24 \mathrm{~h} \text { exposure } \\
\text { Sun dried for } 28 \text { days }\end{array}$ \\
\hline
\end{tabular}

Table 7. Mix quantities for GPC made with FA and GGBS

\begin{tabular}{|c|c|c|c|c|c|c|c|}
\hline \multirow{2}{*}{$\begin{array}{l}\text { GPC } \\
\text { Mix }\end{array}$} & \multicolumn{2}{|c|}{$\operatorname{AAS}\left(\mathrm{kg} / \mathrm{m}^{3}\right)$} & \multirow{2}{*}{$\begin{array}{l}\text { Fly ash } \\
\left(\mathrm{kg} / \mathrm{m}^{3}\right)\end{array}$} & \multirow{2}{*}{$\begin{array}{c}\text { GGBS } \\
\left(\mathrm{kg} / \mathrm{m}^{3}\right)\end{array}$} & \multirow{2}{*}{$\begin{array}{c}\text { Fine } \\
\text { Aggregate } \\
\left(\mathrm{kg} / \mathrm{m}^{3}\right)\end{array}$} & \multirow{2}{*}{$\begin{array}{c}\text { Coarse } \\
\text { Aggregate } \\
\left(\mathrm{kg} / \mathrm{m}^{3}\right)\end{array}$} & \multirow{2}{*}{ Curing } \\
\hline & $\mathrm{NaOH}$ & $\mathrm{Na}_{2} \mathrm{SiO}_{3}$ & & & & & \\
\hline GP7 & 64.29 & 160.71 & 350 & 100 & 505 & 1246 & $\begin{array}{c}\text { Heat @ } 60{ }^{\circ} \mathrm{C} \text { for } 24 \mathrm{~h} \text { exposure/ } \\
\text { Sun dried for } 28 \text { days }\end{array}$ \\
\hline GP8 & 64.29 & 160.71 & 350 & 100 & 505 & 1246 & $\begin{array}{c}\text { Heat @ } 60{ }^{\circ} \mathrm{C} \text { for } 24 \mathrm{~h} \text { exposure/ } \\
\text { Sun dried for } 28 \text { days }\end{array}$ \\
\hline GP9 & 64.29 & 160.71 & 350 & 100 & 505 & 1246 & $\begin{array}{c}\text { Heat @ } 60{ }^{\circ} \mathrm{C} \text { for } 24 \mathrm{~h} \text { exposure/ } \\
\text { Sun dried for } 28 \text { days }\end{array}$ \\
\hline GP10 & 64.29 & 160.71 & 350 & 100 & 505 & 1246 & $\begin{array}{c}\text { Heat @ } 60{ }^{\circ} \mathrm{C} \text { for } 24 \mathrm{~h} \text { exposure/ } \\
\text { Sun dried for } 28 \text { days }\end{array}$ \\
\hline GP11 & 64.29 & 160.71 & 350 & 100 & 505 & 1246 & $\begin{array}{c}\text { Heat @ } 60{ }^{\circ} \mathrm{C} \text { for } 24 \mathrm{~h} \text { exposure/ } \\
\text { Sun dried for } 28 \text { days }\end{array}$ \\
\hline GP12 & 64.29 & 160.71 & 350 & 100 & 505 & 1246 & $\begin{array}{c}\text { Heat @ } 60{ }^{\circ} \mathrm{C} \text { for } 24 \mathrm{~h} \text { exposure/ } \\
\text { Sun dried for } 28 \text { days }\end{array}$ \\
\hline
\end{tabular}


Table 6. Mix quantities for GPC made with FA

\begin{tabular}{|c|c|c|c|c|c|c|c|}
\hline \multirow{2}{*}{$\begin{array}{l}\text { GPC } \\
\text { Mix }\end{array}$} & \multicolumn{2}{|c|}{$\operatorname{AAS}\left(\mathrm{kg} / \mathrm{m}^{3}\right)$} & \multirow{2}{*}{$\begin{array}{l}\text { Fly ash } \\
\left(\mathrm{kg} / \mathrm{m}^{3}\right)\end{array}$} & \multirow{2}{*}{$\underset{\left(\mathrm{kg} / \mathrm{m}^{3}\right)}{\mathrm{MK}}$} & \multirow{2}{*}{$\begin{array}{c}\text { Fine } \\
\text { Aggregate } \\
\left(\mathrm{kg} / \mathrm{m}^{3}\right)\end{array}$} & \multirow{2}{*}{$\begin{array}{c}\text { Coarse } \\
\text { Aggregate } \\
\left(\mathrm{kg} / \mathrm{m}^{3}\right)\end{array}$} & \multirow{2}{*}{ Curing } \\
\hline & $\mathrm{NaOH}$ & $\mathrm{Na}_{2} \mathrm{SiO}_{3}$ & & & & & \\
\hline GP13 & 64.29 & 160.71 & 225 & 225 & 505 & 1246 & $\begin{array}{c}\text { Heat @ } 60{ }^{\circ} \mathrm{C} \text { for } 24 \mathrm{~h} \text { exposure/ } \\
\text { Sun dried for } 28 \text { days }\end{array}$ \\
\hline GP14 & 64.29 & 160.71 & 225 & 225 & 505 & 1246 & $\begin{array}{c}\text { Heat @ } 60{ }^{\circ} \mathrm{C} \text { for } 24 \mathrm{~h} \text { exposure/ } \\
\text { Sun dried for } 28 \text { days }\end{array}$ \\
\hline GP15 & 64.29 & 160.71 & 225 & 225 & 505 & 1246 & $\begin{array}{c}\text { Heat @ } 60{ }^{\circ} \mathrm{C} \text { for } 24 \mathrm{~h} \text { exposure/ } \\
\text { Sun dried for } 28 \text { days }\end{array}$ \\
\hline GP16 & 64.29 & 160.71 & 225 & 225 & 505 & 1246 & $\begin{array}{c}\text { Heat @ } 60{ }^{\circ} \mathrm{C} \text { for } 24 \mathrm{~h} \text { exposure/ } \\
\text { Sun dried for } 28 \text { days }\end{array}$ \\
\hline GP17 & 64.29 & 160.71 & 225 & 225 & 505 & 1246 & $\begin{array}{c}\text { Heat @ } 60{ }^{\circ} \mathrm{C} \text { for } 24 \mathrm{~h} \text { exposure/ } \\
\text { Sun dried for } 28 \text { days }\end{array}$ \\
\hline GP18 & 64.29 & 160.71 & 225 & 225 & 505 & 1246 & $\begin{array}{c}\text { Heat @ } 60{ }^{\circ} \mathrm{C} \text { for } 24 \mathrm{~h} \text { exposure/ } \\
\text { Sun dried for } 28 \text { days }\end{array}$ \\
\hline
\end{tabular}

Table 6. Strengths for GPC made with FA, FA+GGBS and FA+MK

\begin{tabular}{|c|c|c|c|c|c|c|}
\hline \multicolumn{2}{|c|}{ Heat @ $60{ }^{\circ}$ C for 24h exposure } & \multicolumn{3}{c|}{ Sun dried for 28 days } \\
\hline $\begin{array}{c}\text { GPC } \\
\text { Mix }\end{array}$ & $\begin{array}{c}\text { Compressive } \\
\text { Strength } \\
(\mathrm{MPa})\end{array}$ & $\begin{array}{c}\text { Split-tensile } \\
\text { Strength } \\
(\mathrm{MPa})\end{array}$ & $\begin{array}{c}\text { Flexural } \\
\text { Strength } \\
(\mathrm{MPa})\end{array}$ & $\begin{array}{c}\text { Compressive } \\
\text { Strength } \\
(\mathrm{MPa})\end{array}$ & $\begin{array}{c}\text { Split-tensile } \\
\text { Strength } \\
(\mathrm{MPa})\end{array}$ & $\begin{array}{c}\text { Flexural } \\
\text { Strength } \\
(\mathrm{MPa})\end{array}$ \\
\hline GP1 & 25.52 & 2.30 & 3.54 & 21.69 & 1.96 & 3.01 \\
\hline GP2 & 27.80 & 2.50 & 3.69 & 23.63 & 2.13 & 3.14 \\
\hline GP3 & 29.07 & 2.62 & 3.77 & 24.71 & 2.23 & 3.20 \\
\hline GP4 & 29.68 & 2.67 & 3.81 & 25.23 & 2.27 & 3.24 \\
\hline GP5 & 39.23 & 3.53 & 4.38 & 33.35 & 3.00 & 3.72 \\
\hline GP6 & 33.35 & 3.00 & 4.04 & 28.35 & 2.55 & 3.43 \\
\hline GP7 & 28.07 & 2.53 & 3.71 & 25.26 & 2.28 & 3.34 \\
\hline GP8 & 30.58 & 2.75 & 3.87 & 27.52 & 2.48 & 3.48 \\
\hline GP9 & 31.98 & 2.88 & 3.96 & 28.78 & 2.59 & 3.56 \\
\hline GP10 & 32.65 & 2.94 & 4.00 & 29.39 & 2.65 & 3.60 \\
\hline GP11 & 43.15 & 3.88 & 4.60 & 38.84 & 3.49 & 4.14 \\
\hline GP12 & 36.69 & 3.30 & 4.24 & 33.02 & 2.97 & 3.82 \\
\hline GP13 & 31.90 & 2.87 & 3.95 & 31.26 & 2.81 & 3.87 \\
\hline GP14 & 34.75 & 3.13 & 4.13 & 34.06 & 3.07 & 4.05 \\
\hline GP15 & 36.34 & 3.27 & 4.22 & 35.61 & 3.20 & 4.14 \\
\hline GP16 & 37.10 & 3.34 & 4.26 & 36.36 & 3.27 & 4.17 \\
\hline GP17 & 49.04 & 4.41 & 4.90 & 48.06 & 4.32 & 4.80 \\
\hline GP18 & 41.69 & 3.75 & 4.52 & 40.86 & 3.68 & 4.43 \\
\hline
\end{tabular}




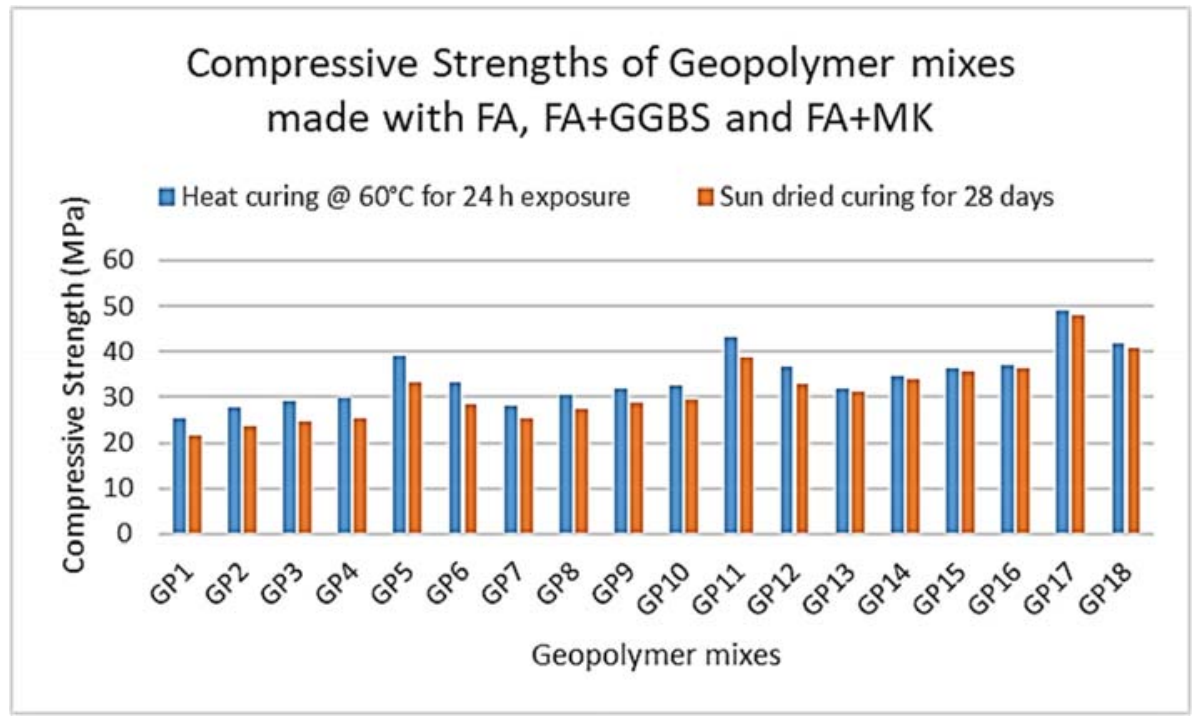

Fig.1. Compressive Strengths for GPC made with FA, FA+GGBS and FA+MK

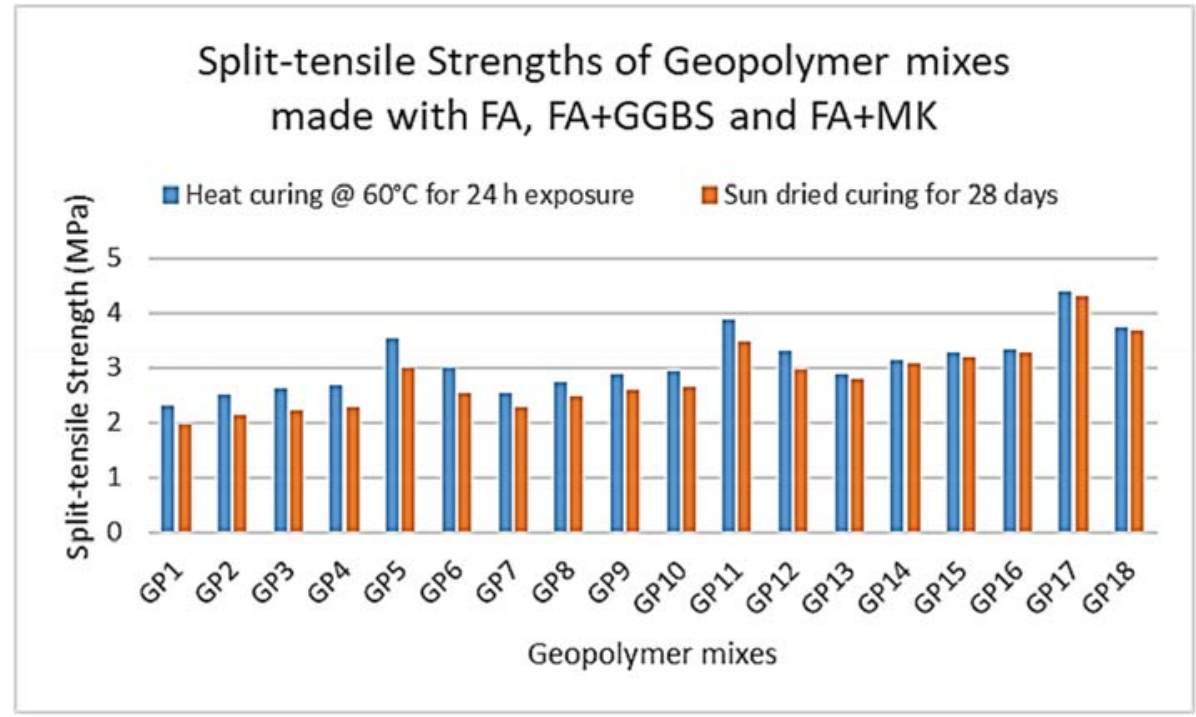

Fig.2. Split-tensile Strengths for GPC made with FA, FA+GGBS and FA+MK 


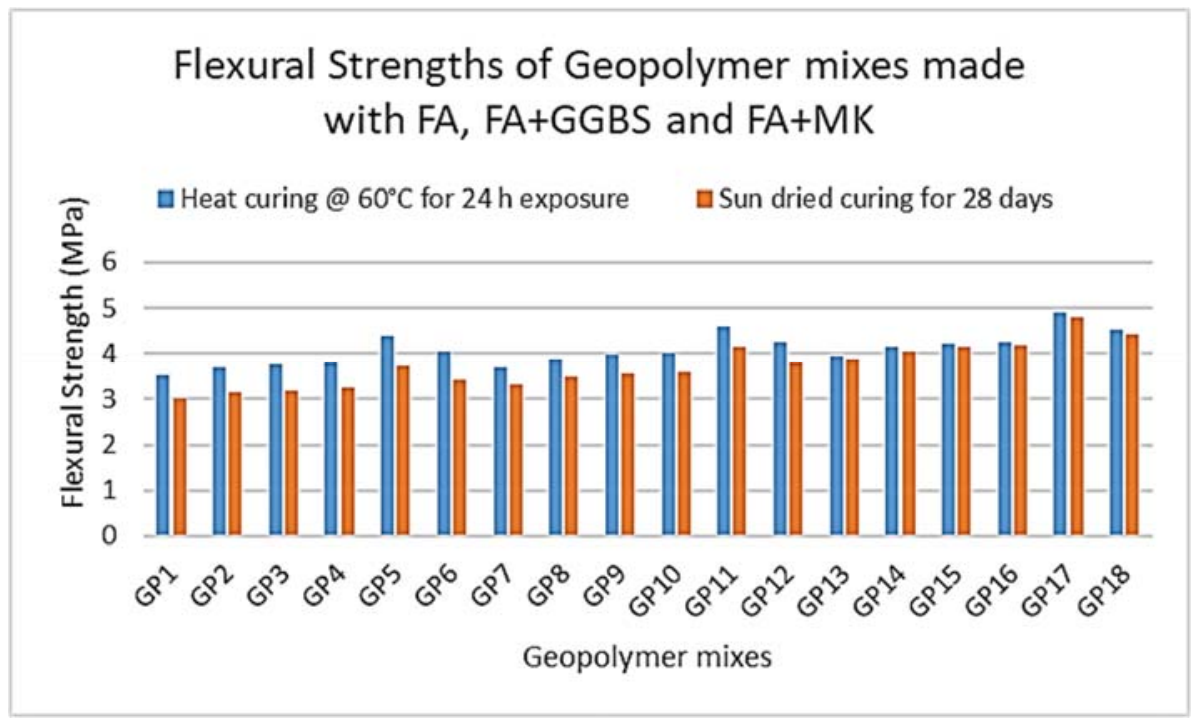

Fig.3. Flexural Strengths for GPC made with FA, FA+GGBS and FA+MK

\section{Conclusions}

1. Geopolymer concrete made with FA produces calcium aluminosilicate hydrate (C-A-S-H) product due to presence of alumina and sodium aluminosilicate hydrate $(\mathrm{N}-\mathrm{A}-\mathrm{S}-\mathrm{H})$ gel as main reaction product of polymerization

2. Geopolymer concrete made with FA and GGBS, calcium silicate hydrate (C-S-H) also gets produced additionally with calcium aluminosilicate hydrate (C-A-S$\mathrm{H})$ gel and sodium aluminosilicate hydrate (N-A-S-H) gel due to presence of high content of $\mathrm{CaO}$ in GGBS. This additional product imparts more strength performance in GPC

3. In geopolymer concrete made with FA and MK, the more amount of calcium aluminosilicate hydrate (C-A-S-H) is produced due to presence of high amount of alumina in metakaolin along with sodium aluminosilicate hydrates (N-A-S$\mathrm{H})$ giving more strength to GPC.

4. Metakaolin is recommended to be used for the development of GPC because it has high amount of alumina.

\section{References}

1. Srinivas. T, Abhignya. $G$ and Ramana Rao. N.V, A Review on Geopolymer RCC Beams made with Recycled Coarse Aggregate, E3S Web of Conferences, ICMED, 10-12 July 2020, India (2020).

2. T. Srinivas, S. V. Srinidhi and N.V. Ramana Rao, A Review on Flexural Behavior of RCC Beams Made with Geopolymer Concrete, E3S
Web of Conferences, ICMED, 10-12 July 2020, India (2020).

3. T. Srinivas , P. Bhavana, and N. V. Ramana Rao, Effect of Manufactured Sand on Flexural Behavior of Geopolymer RCC Beams: A review, E3S Web of Conferences, ICMED, 10-12 July 2020, India (2020).

4. T. Srinivas and N.V.Ramana Rao, IJCIET, Volume 10, 510 (2019).

5. K. Sai Gopi, Dr. T. Srinivas and S. P. Raju V, E3S Web of Conferences ICMED 184, 01084GRIET, 28-29 February, https://doi.org/10.1051/e3sconf/20201840110 84(2020)

6. Jagannadha Kumar, M.V., Jagannadha Rao, K., Dean Kumar, B., Srinivasa Reddy, V., Int. J. of Civil Eng. and Tech., 9(7), pp. 11331141 (2018)

7. M. Kavitha, P. B. Bobba and D. Prasad, 2016 IEEE 7th Power India International Conference (PIICON), 2016, pp. 1-6

8. Ganta, J.K., Seshagiri Rao, M.V., Mousavi, S.S., Srinivasa Reddy, V., Bhojaraju, C., Structures 28, pp. 956-972 (2020)

9. Naidu, K.S.S.T., Rao, M.V.S., Reddy, V.S., Int. J. of Innov. Tech. and Explor. Eng.g (IJITEE), 8(9 Special Issue 2), pp. 641-642 (2019)

10. A.U. Haq, A. K. Kavit, T. Rao, T. Buddi, D. Baloji, K. Satyanarayana, S. K. Singh, Materials Today: Proceedings, 18, 4589 (2019)

11. Chandana Priya, C., Seshagiri Rao, M.V., Srinivasa Reddy, V., Int. J. of Civil Eng. and Tech., 9(11), pp. 2218-2225 (2018)

12. Satya Sai Trimurty Naidu, K., Seshagiri Rao, M.V., Srinivasa Reddy, V., Int. J. of Civil Eng. and Tech., 9(11), pp. 2383-2393 (2018)

13. Supriya, Y., Srinivasa Reddy, V., Seshagiri 
Rao, M.V., Shrihari, S., Int. J. of Rec. Tech. and Engi., 8(3), pp. 5381-5385 (2019)

14. Kotkunde, N., Krishna, G., Shenoy, S.K., Gupta, A.K., Singh, S.K. International Journal of Material Forming, 10 (2), pp. 255-266 (2017)

15. M. Vaishnavi and P. B. Bobba, 2019 IEEE 5th International Conference for Convergence in Technology (I2CT), 2019, pp. 1-6

16. Govardhan, D., Kumar, A.C.S., Murti, K.G.K., Madhusudhan Reddy, G. Materials and Design, 36, pp. 206-214. (2012)

17. Kumar, P., Singhal, A., Mehta, S., Mittal, A.
Journal of Real-Time Image Processing, 11 (1), pp. 93-109. (2016)

18. Raghunadha Reddy, T., Vishnu Vardhan, B., Vijayapal Reddy, P. International Journal of Applied Engineering Research, 11 (5), pp. 3092-3102 (2016)

19. Srinivas Rao J, S K Tummala, Kuthuri N R, Indonesia Journal of Electrical Engg. \& Computer Science, 21 (723), 2020

20. Hussaini, S.M., Krishna, G., Gupta, A.K., Singh, S.K. Journal of Manufacturing Processes, 18, pp. 151-158 (2015) 